Virginia Commonwealth University VCU Scholars Compass

2006

\title{
Subpicosecond time-resolved Raman studies of field-induced transient transport in an InxGa 1 -xAs-based p-i-n semiconductor nanostructure
}

\author{
K.T.Tsen
}

Arizona State University at the Tempe Campus, tsen@asu.edu

Juliann G. Kiang

Uniformed Services University of the Health Sciences

D. K. Ferry

Arizona State University at the Tempe Campus

Hadis Morkoç

Virginia Commonwealth University, hmorkoc@vcu.edu

Follow this and additional works at: http://scholarscompass.vcu.edu/phys_pubs

Part of the Physics Commons

Tsen, K. T., Kiang, J. G., Ferry, D. K., et al. Subpicosecond time-resolved Raman studies of field-induced transient transport in an InxGa 1-xAs-based p-i-n semiconductor nanostructure. Applied Physics Letters, 89, 262101 (2006).

Copyright (C) 2006 AIP Publishing LLC.

\section{Downloaded from}

http://scholarscompass.vcu.edu/phys_pubs/36

This Article is brought to you for free and open access by the Dept. of Physics at VCU Scholars Compass. It has been accepted for inclusion in Physics Publications by an authorized administrator of VCU Scholars Compass. For more information, please contact libcompass@vcu.edu. 


\title{
Subpicosecond time-resolved Raman studies of field-induced transient transport in an $\ln _{x} \mathrm{Ga}_{1-x} A s$-based $p$-i-n semiconductor nanostructure
}

\author{
K. T. Tsen ${ }^{\text {a) }}$ \\ Department of Physics and Astronomy, Arizona State University, Tempe, Arizona 85287 \\ Juliann G. Kiang \\ Armed Forces Radiobiology Research Institute, Uniformed Services University of the Health Sciences, \\ Bethesda, Maryland 20889 \\ D. K. Ferry \\ Department of Electrical Engineering, Arizona State University, Tempe, Arizona 85287 \\ H. Morkoç \\ Department of Physics and Electrical Engineering, Virginia Commonwealth University, Richmond, Virginia \\ 23284
}

(Received 21 August 2006; accepted 15 November 2006; published online 26 December 2006)

\begin{abstract}
Electron transient transport in an $\operatorname{In}_{x} \mathrm{Ga}_{1-x}$ As-based $(x=0.53) p-i-n$ nanostructure under the application of an electric field has been studied by time-resolved Raman spectroscopy on a subpicosecond time scale and at $T=300 \mathrm{~K}$. The experimental results reveal the time evolution of the electron distribution function and electron drift velocity with subpicosecond time resolution. These experimental results are compared with those of both InP-based and GaAs-based $p$ - $i-n$ nanostructures and provide a consistent understanding and better insight of electron transient transport phenomena in semiconductors. (c) 2006 American Institute of Physics.
\end{abstract}

[DOI: $10.1063 / 1.2420782$ ]

Recently, electronic devices with sizes of the order of $0.1 \mu \mathrm{m}$ or smaller have become achievable. This capability, when coupled with a typical device operation voltage (which is of the order of $1 \mathrm{~V}$ ), suggests that carrier transient effects may be the dominant transport properties for electrons or holes in semiconductor nanostructures.

Many theoretical (mainly, Monte Carlo simulations) and experimental efforts ${ }^{1-15}$ have been made to investigate these interesting yet challenging transient carrier transport phenomena in semiconductors. Recently, Grann et al. ${ }^{16-20}$ have investigated nonequilibrium electron distributions, electron drift velocities, and nonequilibrium longitudinal optical phonon populations in a GaAs-based $p-i-n$ nanostructure by using picosecond/subpicosecond transient/time-resolved Raman spectroscopy. These authors have directly confirmed the existence of electron velocity overshoot phenomenon in semiconductors. In spite of tremendous efforts on the study of carrier transient transport phenomena, lack of a better insight for these transient electron transport phenomena still prevails. In this letter, subpicosecond time-resolved Raman spectroscopy has been used for probing electron transient phenomena in an $\operatorname{In}_{0.53} \mathrm{Ga}_{0.47}$ As-based $p$ - $i-n$ nanostructure. Our experimental results reveal the time evolution of the electron distribution as well as the electron drift velocity with subpicosecond time resolution. The detailed structure of the $\mathrm{In}_{0.53} \mathrm{Ga}_{0.47} \mathrm{As}$-based nanostructure used in this work has been shown elsewhere. ${ }^{21}$ The $\mathrm{Zn}$-doped $p$-type layer and $\mathrm{Si}$ doped $n$-type layer together serve as a capacitor and provide a uniform electric field across the active region of the sample. We estimate the capacitance in our sample configuration to be $C \cong 2.6 \times 10^{-11} \mathrm{~F}$. Since the resistance of the sample is $R \cong 100 \mathrm{k} \Omega$, with such a long $R C$ time constant the

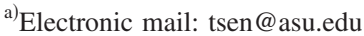

capacitance of the sample structure will not affect our subpicosecond measurements in any way.

The sample is excited and probed by the outputs of two optical parametric amplifiers (OPA1 and OPA2) (Ref. 22) pumped by a common pulse from a Ti-sapphire amplifier system which is composed of the ultrastable Millennia/ Tsunami short pulse oscillator and the Spitfire regenerative amplifier with the Merlin Nd:YLF pump laser. The output from OPA1 has a pulse width of about $100 \mathrm{fs}$ (full width at half maximum) and is chosen to operate at photon energy of $\hbar \omega_{\text {pump }} \cong 1.05 \mathrm{eV}$. We estimate the absorption depth of the sample at such excitation laser photon energy to be about $6500 \AA$. This is used to excite electron-hole pairs in the $\mathrm{In}_{0.53} \mathrm{Ga}_{0.47}$ As-based $p$ - $i-n$ semiconductor nanostructure. The output from OPA2 has the same pulse width and is used to probe the electron distributions with photon energy of $\hbar \omega_{\text {probe }} \cong 0.85 \mathrm{eV}$. This experimental arrangement ensures that negligible electrons escape from the probe region during the time-resolved Raman measurements. The photoexcited electron-hole pair density was estimated from the average laser power, focused spot size on the sample surface, and the absorption depth at the excitation laser wavelength. The backscattered Raman signals were collected and analyzed by a standard computer-controlled Raman system, which includes a double spectrometer, a photomultiplier tube, and associated photon counting electronics. All the data were taken at $T=300 \mathrm{~K}$. The single-particle scattering experiments, which were used to measure directly the electron distributions, were conducted in the $Z(X, Y) \bar{Z}$ scattering geometry, where $X=(100), Y=(010)$, and $Z=(001)$. This scattering configuration ensures the detection of a scattered light signal from only spin-density fluctuations. ${ }^{23,24}$ The effective electric field intensity was determined by using the Franz-Keldysh effect, as demonstrated in Ref. 25. The nonequilibrium elec- 

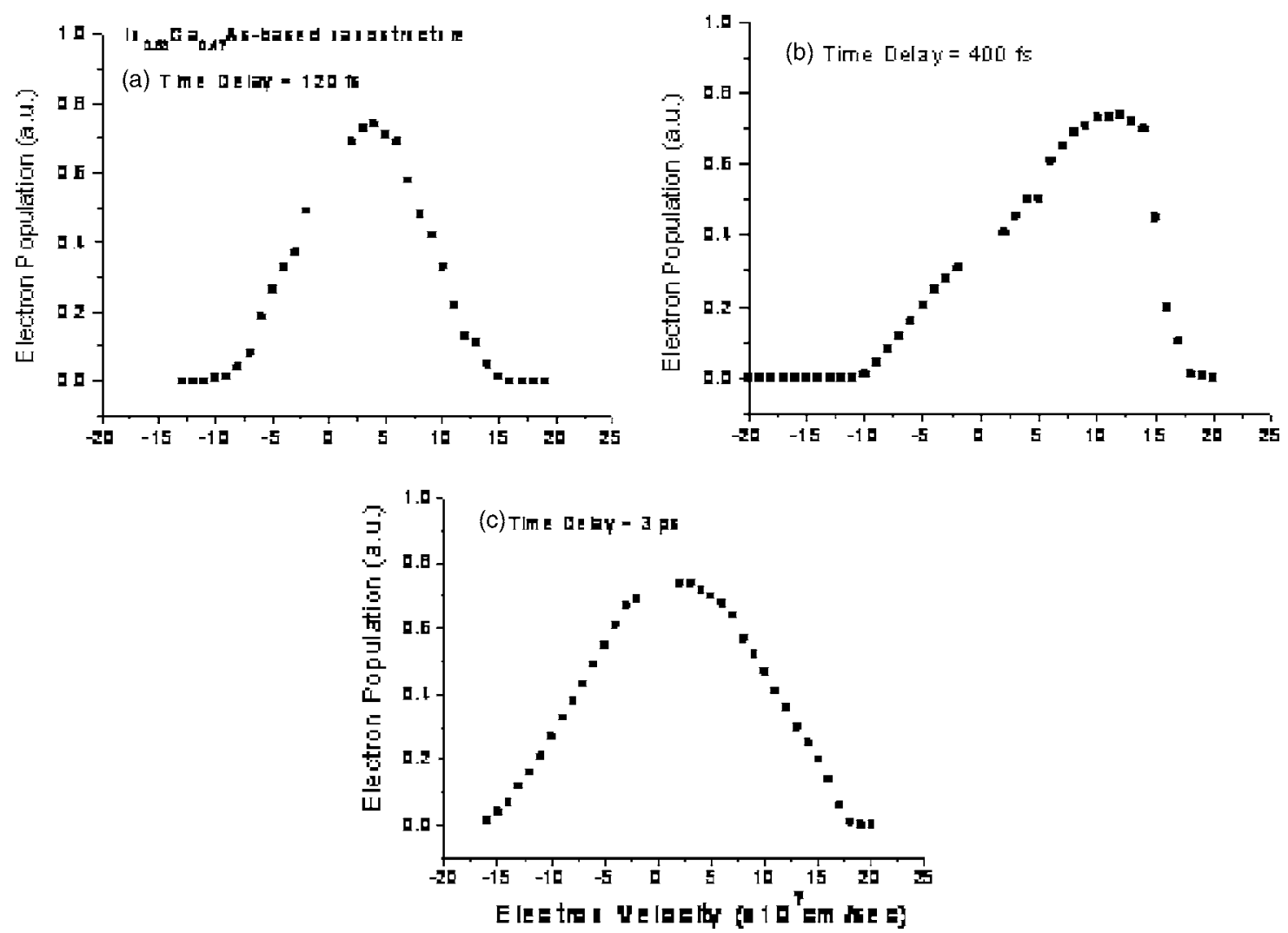

FIG. 1. Nonequilibrium electron distribution for an $\operatorname{In}_{0.53} \mathrm{Ga}_{0.47} \mathrm{As}$-based $p-i-n$ nanostructure measured at an electric field intensity $E=20 \mathrm{kV} / \mathrm{cm}$, a photoexcited electron-hole pair density $n \cong 5 \times 10^{16} \mathrm{~cm}^{-3}$, and for time delays of (a) $120 \mathrm{fs}$, (b) $400 \mathrm{fs}$, and (c) 3 ps. The time evolution of electron distributions provides a better insight of electron transient transport phenomena in semiconductors.

tron distributions are directly measured in a way similar to Ref. 20.

A typical nonequilibrium electron distribution for an $\mathrm{In}_{0.53} \mathrm{Ga}_{0.47} \mathrm{As}$-based nanostructure, taken at an electric field intensity $E=20 \mathrm{kV} / \mathrm{cm}$, with electron-hole pair density $n$ $\cong 5 \times 10^{16} \mathrm{~cm}^{-3}$ and at a time delay $\Delta t=400$ fs between the pump and probe pulses, is shown in Fig. 1(b). We have found that the electron distribution shifts toward the $-\mathbf{E}$ direction, as expected. The electron distribution function cannot be fitted with either a shifted Fermi-Dirac or a MaxwellBoltzmann distribution, suggesting that electron distribution during the transient is in an extremely nonequilibrium state. A sharp cutoff in the electron velocity near $1.5 \times 10^{8} \mathrm{~cm} / \mathrm{s}$ is observed, indicative of both the onset of electron intervalley scattering processes and the velocity saturation due to the nonparabolic effective mass. We note that impact ionization has a minimal effect on the cutoff velocity because it happens at a time scale of $100 \mathrm{ps}-1 \mathrm{~ns}$. The electron drift velocity in Fig. 1(b) has been found to be $V_{d}=6.9 \pm 0.7$ $\times 10^{7} \mathrm{~cm} / \mathrm{s}$.

Figure 2 shows the electron drift velocity as a function of the time delay for an $\operatorname{In}_{0.53} \mathrm{Ga}_{0.47}$ As-based $p$ - $i$ - $n$ nanostructure, taken at an electric field intensity $E=20 \mathrm{kV} / \mathrm{cm}$. This is contrasted with equivalent curves taken in the other two material systems-GaAs and InP. We observe that the electron drift velocity increases linearly with the time delay during the first $200 \mathrm{fs}$, which provides an unambiguous evidence of electron ballistic transport under our experimental conditions. After $200 \mathrm{fs}$, the drift velocity increases sublinearly indicating the onset of various electron scattering processes. It peaks at around $6.9 \times 10^{7} \mathrm{~cm} / \mathrm{s}$ at $\Delta t=400 \mathrm{fs}$, and then decreases gradually toward its steady-state value of about
$3.0 \times 10^{7} \mathrm{~cm} / \mathrm{s}$. This latter value is also inferred from ensemble Monte Carlo studies of bulk $\operatorname{In}_{x} \mathrm{Ga}_{1-x} \mathrm{As}$ at this composition.

We have also performed similar experiments on a GaAsbased $p-i-n$ and an InP-based $p-i-n$ nanostructure, in which we kept the electron excess energy the same, i.e., $\Delta E$ $\cong 0.30 \mathrm{eV}$. These results are also shown in Fig. 2. There are three interesting features that are worthwhile pointing out. (1) The peak value of electron drift velocity is significantly larger for $\mathrm{In}_{0.53} \mathrm{Ga}_{0.47} \mathrm{As}$ than for either GaAs or InP, and it occurs at a later time (400 fs for $\operatorname{In}_{0.53} \mathrm{Ga}_{0.47} \mathrm{As}$ compared

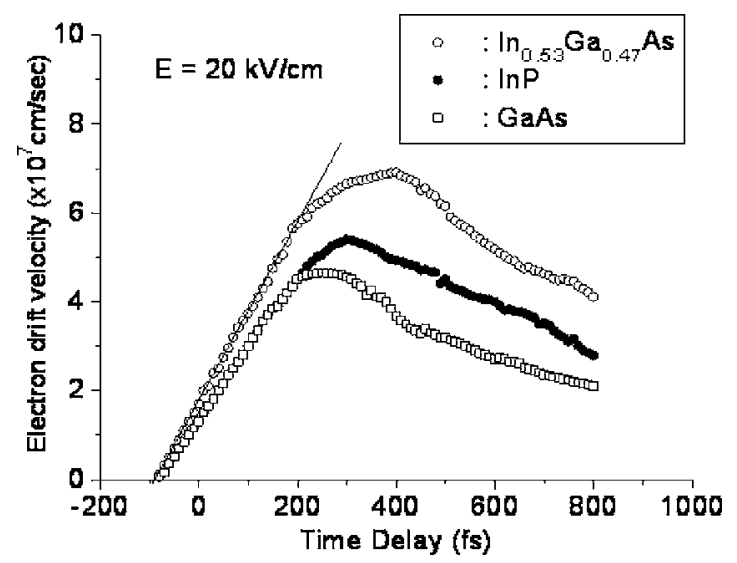

FIG. 2. Electron drift velocity as a function of time delay for an $\mathrm{In}_{0.53} \mathrm{Ga}_{0.47} \mathrm{As}$-based $p-i-n$ nanostructure (open circles), an InP-based $p-i-n$ nanostructure (solid circles), and a GaAs-based $p$ - $i-n$ nanostructure (open squares). The straight line drawn between $\Delta t=0$ and $200 \mathrm{fs}$ on the data of an $\mathrm{In}_{0.53} \mathrm{Ga}_{0.47}$ As-based $p-i-n$ nanostructure indicates the time interval during which electrons travel ballistically. 
with $300 \mathrm{fs}$ for $\mathrm{InP}$ and $250 \mathrm{fs}$ for GaAs). This is partly because of the smaller electron effective mass and partly because of larger energy separation between $\Gamma$ and $X$ valleys for $\mathrm{In}_{0.53} \mathrm{Ga}_{0.47} \mathrm{As}$. (2) The slope from the ballistic portion of the data is steeper for $\mathrm{In}_{0.53} \mathrm{Ga}_{0.47} \mathrm{As}$ than for $\mathrm{GaAs}$ or $\mathrm{InP}$, which reflects the fact that, even for an energy of about $0.30 \mathrm{eV}$ above the conduction band, $\mathrm{In}_{0.53} \mathrm{Ga}_{0.47} \mathrm{As}$ has a smaller effective mass than either GaAs or InP. (3) The observed maximum electron drift velocity is substantially higher in InP than in GaAs. This is primarily due to the fact that the energy separation between the $\Gamma$ and $X$ valleys is significantly larger in InP than in GaAs. All of these results are consistent with our previous understanding of electron transient transport in these semiconductors.

To gain a better insight of the electron transient phenomena, we have plotted in Fig. 1 the electron distribution for the $\mathrm{In}_{0.53} \mathrm{Ga}_{0.47} \mathrm{As}$ nanostructure, taken at $E=20 \mathrm{kV} / \mathrm{cm}$ and at a variety of time delays: $\Delta t=120 \mathrm{fs}, 400 \mathrm{fs}$, and $3 \mathrm{ps}$, as indicated. For $\Delta t=120 \mathrm{fs}$, the electron distribution resembles a symmetric, shifted distribution, suggesting that electrons have been accelerated and moved rigidly for the same amount in velocity space, which is consistent with our observation in Fig. 2 that electrons travel ballistically during the first $200 \mathrm{fs}$. As a matter of fact, for time delay between 0 and $200 \mathrm{fs}$, the electron drift velocity is linearly proportional to the elapsed time and to the magnitude of applied electric field. This is usually referred to as "electron ballistic transport." As the time delay progressively increases to $\Delta t$ $=400 \mathrm{fs}$, the electron distribution exhibits an extremely nonequilibrium, nonsymmetric nature. At this time delay, some elastic as well as inelastic electron scattering processes become effective. The electron drift velocity can keep increasing with the time delay but it can no longer increase linearly because these electron scattering processes efficiently randomize electron momentum and reduce the electron drift velocity. Nevertheless, if the electric field intensity is sufficiently large, the acquired electron drift velocity during this time interval can be substantially larger than its steady-state value. This is manifested in the time delay between 200 and $400 \mathrm{fs}$ in Fig. 2. This is commonly called "electron velocity overshoot." When the time delay increases even further to $\Delta t=3 \mathrm{ps}$, the electron distribution has been found to be approximately symmetric but significantly broader than that at $\Delta t=120 \mathrm{fs}$. This is because at this long time delay all of the elastic and inelastic electron scattering processes become operative. The electron distribution spreads out and the electron drift velocity gradually approaches its steady-state value.

We note that the temperature will have only a small effect on our results, while the electric field strength is the important quantity, transient transport does not occur in a small field, and the overshoot is much more pronounced in a large field.

In conclusion, we have used subpicosecond timeresolved Raman spectroscopy to interrogate electron transient transport in an $\mathrm{In}_{0.53} \mathrm{Ga}_{0.47}$ As-based $p-i$ - $n$ semiconduc- tor nanostructure under the application of an electric field. Our experimental results reveal the time evolution of the electron distribution function and the electron drift velocity with subpicosecond time resolution. These experimental results are compared with those of InP-based $p-i-n$ and GaAsbased $p-i-n$ nanostructures and provide a consistent understanding and better insight of electron transient transport phenomena in semiconductors.

This work is supported by the National Science Foundation under Grant No. DMR-0305147.

${ }^{1}$ D. K. Ferry, H. L. Grubin, and G. J. Iafrate, in Semiconductors Probed by Ultrafast Laser Spectroscopy, edited by R. R. Alfano (Academic, New York, 1984), Vol. 1, p. 413.

${ }^{2}$ E. Constant, in Hot Electron Transport in Semiconductors, Topics in Applied Physics Vol. 58, edited by L. Reggiani (Springer, Berlin, 1985), p. 227.

${ }^{3}$ J. Shah and R. F. Leheny, in Semiconductors Probed by Ultrafast Laser Spectroscopy (Ref. 1), p. 45.

${ }^{4} \mathrm{~K}$. T. Tsen, in Ultrafast Phenomena in Semiconductors, edited by K. T. Tsen (Springer, New York, 2001), p. 191.

${ }^{5}$ J. G. Ruch, IEEE Trans. Electron Devices ED-19, 652 (1972).

${ }^{6}$ T. J. Maloney and J. Frey, J. Appl. Phys. 48, 781 (1977).

${ }^{7}$ M. S. Shur and L. F. Eastman, Solid-State Electron. 24, 11 (1981).

${ }^{8}$ S. Tanaka, H. Kobayashi, H. Satito, and H. Shionoya, J. Phys. Soc. Jpn. 49, 1051 (1980)

${ }^{9}$ W. Graudszus and E. O. Goebel, J. Phys. Colloq. C7, 437 (1981).

${ }^{10}$ D. Rosen, A. G. Donkas, Y. Bundanky, A. Katy, and R. R. Alfano, Appl. Phys. Lett. 39, 935 (1981).

${ }^{11}$ W. Sha, T. B. Norris, W. J. Schaff, and K. E. Meyer, Phys. Rev. Lett. 67, 2553 (1991).

${ }^{12}$ C. V. Shank, R. L. Fork, B. I. Greene, F. K. Reinhard, and R. A. Logan, Appl. Phys. Lett. 38, 104 (1981).

${ }^{13}$ K. E. Meyer, M. Pessot, G. A. Mourou, R. O. Grondin, and S. N. Chamoun, Appl. Phys. Lett. 53, 2254 (1988).

${ }^{14}$ J. Son, W. Sha, J. Kim, T. B. Norris, J. F. Whitaker, and G. A. Mourou, Appl. Phys. Lett. 63, 923 (1993).

${ }^{15}$ B. B. Hu, E. A. de Souza, W. H. Knox, J. E. Cunningham, M. C. Nuss, A. V. Kuznetsov, and S. L. Chuang, Phys. Rev. Lett. 74, 1689 (1995).

${ }^{16}$ E. D. Grann, S. J. Sheih, C. Chia, K. T. Tsen, O. F. Sankey, S. E. Guncer, D. K. Ferry, G. Maracas, R. Droopad, A. Salvador, A. Botcharev, and H. Morkoc, Appl. Phys. Lett. 64, 1230 (1994).

${ }^{17}$ E. D. Grann, K. T. Tsen, O. F. Sankey, D. K. Ferry, A. Salvador, A. Botcharev, and H. Morkoc, Appl. Phys. Lett. 67, 1760 (1995).

${ }^{18}$ E. D. Grann, S. J. Sheih, K. T. Tsen, O. F. Sankey, S. E. Guncer, D. K. Ferry, A. Salvador, A. Botcharev, and H. Morkoc, Phys. Rev. B 51, 1631 (1995).

${ }^{19}$ E. D. Grann, K. T. Tsen, D. K. Ferry, A. Salvador, A. Botcharev, and H. Morkoc, Phys. Rev. B 53, 9838 (1996).

${ }^{20}$ E. D. Grann, K. T. Tsen, D. K. Ferry, A. Salvador, A. Botcharev, and H. Morkoc, Phys. Rev. B 56, 9539 (1997).

${ }^{21}$ K. T. Tsen, Juliann G. Kiang, D. K. Ferry, V. A. Kochelap, S. M. Komirenko, K. W. Kim, and H. Morkoc, J. Phys.: Condens. Matter 18, 7961 (2006).

${ }^{22}$ K. T. Tsen, in Ultrafast Dynamical Processes in Semiconductors, Topics in Applied Physics Vol. 92, edited by K. T. Tsen (Springer, Heidelberg, 2004), p. 193.

${ }^{23}$ M. V. Klein, in Light Scattering in Solids I, Topics in Applied Physics Vol. 8, edited by M. Cardona (Springer, New York, 1983), p. 151.

${ }^{24}$ G. Abstreiter, M. Cardona, and A. Pinczuk, in Light Scattering in Solids $I V$, edited by M. Cardona and G. Güntherodt (Springer, New York, 1986), p. 5.

${ }^{25}$ K. T. Tsen, R. P. Joshi, A. Salvador, A. Botcharev, and H. Morkoc, J. Appl. Phys. 81, 406 (1997). 